\title{
Prediction of twin pregnancy preeclampsia based on clinical risk factors, early pregnancy serum markers, and uterine artery pulsatility index
}

\author{
Yan $L u^{1}$, Zhongying Ding ${ }^{2}$, Wenwen $\mathrm{Li}^{3}$, \\ Lina $\mathrm{Mei}^{4}$, Linglong Shen ${ }^{5}$, Huaying Shan
}

\begin{abstract}
Objectives: To investigate whether a combination of clinical risk factors, early pregnancy serum markers, and uterine artery pulsatility index (UTPI) can be used to predict twin preeclampsia (PE).

Methods: This case control study included women with twin pregnancies who had undergone obstetrics treatments and gave birth at the Huzhou Maternity and Child Health Care Hospital from October 2018 to November 2020. Patients with PE comprised study group, and patients without PE comprised control group based on selection criteria and a 1:1 ratio. Statistical analysis was performed using clinical risk factors, early pregnancy serum markers, and UTPIs, and the area under the receiver operating curve (AUC. Sensitivity, and the specificity of different combinations of these variables were calculated to predict PE in women with twin pregnancy.

Results: Logistic regression analysis revealed four independent predictors for the onset of PE during twin pregnancies: first delivery (OR, 7.51; $\mathrm{P}=0.045)$, conception method $(\mathrm{OR}, 7.11 ; \mathrm{P}=0.036)$, B-HCG level (per SD OR, 2.73; $P=0.026)$, and UTPI (OR, 0.17; $P=0.043)$. First-delivery and IVF pregnancy methods both lead to a 7 -fold increase in the PE risk during twin pregnancies. Every one sigma (standard deviation) increase in the B-HCG level led to a 2.73 -fold increase in the PE risk. Every UTPI increment by 1.0 reduces the risk of PE by $83 \%$. The prediction efficiencies were based on an AUC of 0.837 , a sensitivity of $69 \%$, and a specificity of $92 \%$ for the clinical risk factors; an AUC of 0.800 , a sensitivity of $81 \%$, and specificity of $78 \%$ for the B-HCG level, and an AUC of 0.814 , a sensitivity of $88 \%$, and a specificity of $65 \%$ for the UTPI. AUC was 0.928 , sensitivity $85 \%$, and a specificity $88 \%$ after applying the three types of indicators together for prediction. Conclusions: By combining early pregnancy serum markers (B-HCG), and UTPI, the predictive value for PE during twin pregnancy is improved together with its sensitivity and specificity.
\end{abstract}

KEYWORDS: Twin pregnancy, Preeclampsia, Prediction.

doi: https://doi.org/10.12669/pjms.37.7.5041

How to cite this:

Lu Y, Ding Z, Li W, Mei L, Shen L, Shan H. Prediction of twin pregnancy preeclampsia based on clinical risk factors, early pregnancy serum markers, and uterine artery pulsatility index. Pak J Med Sci. 2021;37(7):1727-1733. doi: https://doi.org/10.12669/pjms.37.7.5041

This is an Open Access article distributed under the terms of the Creative Commons Attribution License (http://creativecommons.org/licenses/by/3.0), which permits unrestricted use, distribution, and reproduction in any medium, provided the original work is properly cited.

Correspondence:

Dr. Yan Lu,

Department of Obstetrics and Gynecology,

Huzhou Maternity and Child Health Care Hospital,

2 Dong Street, Huzhou 313000,

Zhejiang Province,

P.R. China.

E-mail: lysusan0927@163.com

* Received for Publication:

* Revision Received:

* Revision Accepted:
July 16, 2021

August 26, 2021

September 5, 2021

\section{INTRODUCTION}

Preeclampsia (PE) is a serious late pregnancy complication, characterized by pregnancyinduced hypertension, usually accompanied by abnormal cardiovascular, endocrine, and nervous system changes. PE is associated with adverse maternal and perinatal outcomes, such as severe hypertension, heart failure, premature birth, placental abruption, neonatal ischemic hypoxic encephalopathy, and is the second leading cause 
of maternal and early neonatal mortality in developing countries. ${ }^{1}$ The incidences of twin pregnancies and their complications have increased with the development of assisted reproductive technologies. The risk of PE in women with twin pregnancy is 2-3 times that of women with singleton pregnancy. ${ }^{2}$ Therefore, recent guidelines recommend multi-factorial combined assessment methods to improve the early prediction efficiency for PE. ${ }^{3,4}$ However, most studies on PE prediction have focused on singleton pregnancies and few reports exist on multiple pregnancy PE prediction. We investigated clinical risk factors, serum markers, and uterine artery pulsatility index (UTPI) during early twin pregnancy as predictors of twin pregnancy PE.

\section{METHODS}

Data from women with twin pregnancy with or without PE who gave birth at the Huzhou Maternity and Child Health Care Hospital from October 2018 to November 2020 were collected.

Inclusion Criteria: Twin pregnancies; primiparous or multiparous women; age range of 18-40 years.

Exclusion Criteria: Three or more multiplepregnancies; abnormal uterus; selective fetal reduction; missed prenatal appointments during pregnancy; fetuses with chromosomal or structural abnormalities; unexplained miscarriage or stillbirth; treatment with anticoagulants (aspirin or heparin).

PE diagnostic criteria were based on the ACOG guidelines, and collected according to the case control study principles. ${ }^{3}$ Data from 26 women with twin pregnancies and diagnosed with PE (study group) were matched 1:1 to 26 women without PE (control group). The delivery times for each pair were within two weeks from each other. All women underwent first trimester ultrasound examinations to determine the fetal head and hip diameters, gestational age, fetal neck zona pellucida thickness, and twin chorionicity. All data (including the patient's prenatal examination and hospitalization information) were retrieved from the Zhejiang Provincial Maternity and Child Health System and the electronic medical record system of the hospital, and was used to identify early pregnancy clinical risk factors for PE. The collected information included age, pre-pregnancy body mass index (BMI), conception method (natural conception, artificial insemination, IVF), family history (mother or sister with a history of PE), birth history (pregnancy, parity), and smoking history.

For Uterine artery pulsatility index (UTPI), women from the case study and control groups were examined with ultrasound during the second trimester (ranging from $11+6$ to $13+6$ weeks) by, experienced personnel. Ultrasound machines used included the GE ultrasound Voluson 730, E8, E6 expert, and the GE LOGIQ E9 ultrasound with 5-9 $\mathrm{MHz}$ transvaginal and $3.5 \mathrm{MHz}$ abdominal probes. Women were in a supine position with controlled breathing intensity. After locating the signal from the uterine artery blood flow, the technician manually measured more than three cardiac cycle spectrograms. UTPI value was obtained using an automated computation.

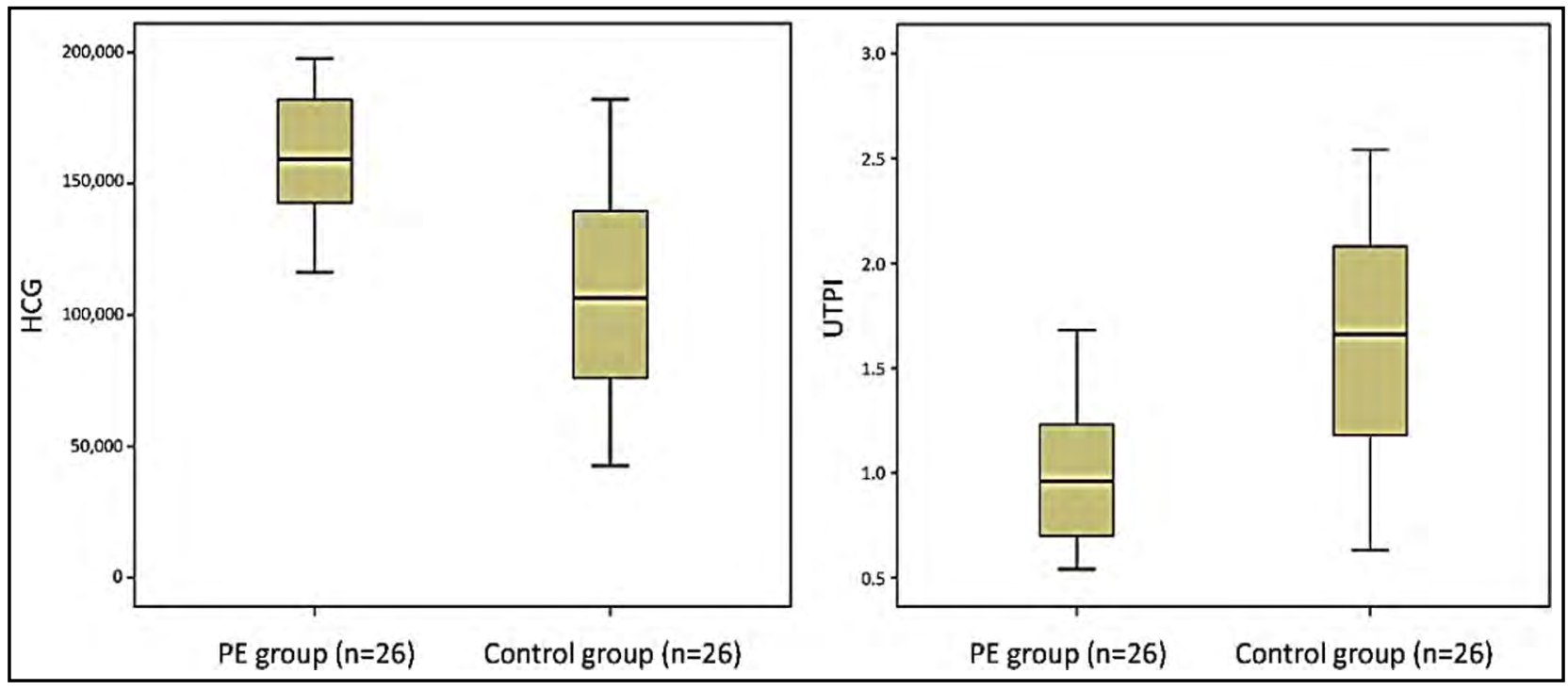

Fig.1: HCG and uterine artery pulsatility index. 
For serum marker detection, $8 \mathrm{ml}$ peripheral blood samples were drawn from all women in the study during the first trimester (between 6 and 14 weeks). Blood supernatants were stored at $-20^{\circ} \mathrm{C}$, and gradually thawed at $4^{\circ} \mathrm{C}$ one day prior to the test. All tests were performed after the diagnosis by personnel with no prior knowledge of the clinical information of the participants. level of $\beta$-human chorionic gonadotropin $(\beta$-HCG) was measured with a PerkinElmer's 1235 time-resolved fluorescence immunoassay and its corresponding hAFP/Free Hcg $\beta$ Dual kit, according to manufacturer's instruction.

A nested case-control study method was used to analyze the association between the clinical risk factors, serum markers, and UTPI values to PE. We drew receiver operating characteristic curves (ROCs) to calculate areas under the curve (AUCs), sensitivity, and specificity, and to evaluate the PE predicting values during twin pregnancies.

All procedures performed in studies involving human participants were in accordance with the ethical standards of the ethics committee of the Huzhou Maternity and Child Health Care Hospital (2019-R-005, 2020-R-007). All patients signed the consent.

Statistical Analysis: Statistical analyses was done using the SPSS20.0 software. Normality and homogeneity of variance tests was applied on measurement data; normally distributed and homogeneous data were represented by means + SDs, and group comparisons were done using $t$-tests. Non-normally distributed data were represented by means and $95 \%$ confidence intervals (CIs), and the Mann-Whitney U-test was used for comparisons between groups. We expressed comparisons of counts with rates, and analyzed them using the $\mathrm{x}^{2}$ or Fisher's exact tests. We performed multivariate logistic regression analysis, drew ROC curves, and calculated areas under the curve (AUC), sensitivities, and specificities. A $\mathrm{P}<0.05$ indicates a statistically significant difference.

\section{RESULTS}

A total of 155 women with twin pregnancy from the first trimester to delivery in our hospital were evaluated for the study. Two women terminated their pregnancies before the 24 weeks of gestation due to fetal developmental abnormalities, and four dropped out of the study due to missing follow-ups. Eventually 26 women developed PE, for an incidence rate of PE during twin pregnancy of $17 \%$. Univariate analysis indicated that the patients with PE had higher average age, higher rate of IVF conception, and earlier gestational weeks of delivery than the women in the control group, with statistically significant differences $(\mathrm{P}<0.05)$. BMI values, pregnancy times, parity, chorionic properties, family PE history, and smoking history were all similar between groups $(\mathrm{P}>0.05)$, Table-I.

Mean $\beta$-HCG level of women with PE was significantly higher than that of the control group,

Table-I: Comparison of the general conditions of the two groups of pregnant women.

\begin{tabular}{|c|c|c|c|c|}
\hline Variable & $\begin{array}{l}\text { Twin pe } \\
(n=26)\end{array}$ & $\begin{array}{c}\text { Control } \\
\text { group } \\
(n=26)\end{array}$ & $X 2 / t$ & $\begin{array}{c}P- \\
\text { value }\end{array}$ \\
\hline Age (years) & & & 10.833 & 0.001 \\
\hline $20-34$ & $15(57.7)$ & $25(96.2)$ & & \\
\hline $35-40$ & $11(42.3)$ & $1(3.8)$ & & \\
\hline $\mathrm{BMI}(\mathrm{kg} / \mathrm{m} 2)$ & & & 0.977 & 0.738 \\
\hline$<18.5$ & $4(15.4)$ & $5(19.2)$ & & \\
\hline$<25$ & $14(53.8)$ & $16(61.5)$ & & \\
\hline$\geq 25$ & $8(30.7)$ & $5(19.2)$ & & \\
\hline Pregnancy times & & & 0.316 & 0.854 \\
\hline 1 & $11(42.3)$ & $13(50.0)$ & & \\
\hline 2 & $9(34.6)$ & $8(30.8)$ & & \\
\hline$\geq 3$ & $6(23.1)$ & $5(19.2)$ & & \\
\hline parity & & & 2.564 & 0.109 \\
\hline 0 & $22(84.6)$ & $17(65.4)$ & & \\
\hline$\geq 1$ & $4(15.4)$ & $9(34.6)$ & & \\
\hline \multicolumn{2}{|c|}{ Conception method } & & 8.571 & 0.014 \\
\hline $\begin{array}{l}\text { Natural } \\
\text { pregnancy }\end{array}$ & $9(34.62)$ & 19(73.1) & & \\
\hline Ovulation & $2(7.7)$ & $2(7.7)$ & & \\
\hline IVF & $15(57.7)$ & $5(19.2)$ & & \\
\hline \multicolumn{2}{|c|}{ Fetal chorionic properties } & & 2.185 & 0.139 \\
\hline $\begin{array}{l}\text { Single cashmere } \\
\text { twins }\end{array}$ & $6(23.1)$ & $11(42.3)$ & & \\
\hline $\begin{array}{l}\text { Double } \\
\text { cashmere twins }\end{array}$ & $20(76.9)$ & $15(57.7)$ & & \\
\hline Family PE history & & & 0.354 & 0.5 \\
\hline Yes & $2(7.7)$ & $1(3.8)$ & & \\
\hline No & $24(92.3)$ & $25(96.2)$ & & \\
\hline Smoking & & & 1.02 & 0.5 \\
\hline Yes & $0(0)$ & 1(3.8) & & \\
\hline No & $26(100)$ & $25(96.2)$ & & \\
\hline $\begin{array}{l}\text { Gestational } \\
\text { week of delivery }\end{array}$ & $\begin{array}{l}35.8 \pm 1.6 \\
\text { (weeks) }\end{array}$ & $36.8 \pm 1.8$ & 17.245 & 0.001 \\
\hline
\end{tabular}

Note: Age, BMI, times of pregnancy, parity, method of conception, nature of fetal chorion, family PE history, smoking cases are expressed as percentages (\%), and gestational week of delivery is expressed as $x \pm s d$. 
Table-II: Comparison of serum markers and UTPI between the two groups.

\begin{tabular}{lccc}
\hline Group & $n$ & $\beta-H C G(\mathrm{mIU} / \mathrm{ml})$ & UTPI \\
\hline PE group & 26 & $161251.77 \pm 22533.35$ & $0.98 \pm 0.32$ \\
Control group & 26 & $104822.85 \pm 38857.63$ & $1.63 \pm 0.60$ \\
$\mathrm{t}$ & & 6.406 & 4.695 \\
P value & & $<0.001$ & $<0.001$ \\
\hline
\end{tabular}

Note: Data is expressed as $\mathrm{x} \pm \mathrm{sd}$.

and the mean UTPI level was lower than that of the control group $(\mathrm{P}<0.05)$. Fig.1 and Table-II.

Taking the occurrence of $\mathrm{PE}$ as the dependent variable (non-PE twins=0, PE twins=1), age, parity, serum markers ( $\beta$-HCG), and UTPI were used as independent variables. We assigned values to the independent variables (age, 2034 years $=1$, 35-39 years $=2$; parity, 0 times $=1, \geq 1$ times=2). The logistic multivariate analysis results showed that the first delivery [(odds ratio (OR), 7.51; 95\% CI, 1.04 to 54.18; $\mathrm{P}=0.045]$, conception method (OR, 7.11; 95\% CI, 1.14 to 44.36 ; $\mathrm{P}=0.036$ ), and $\beta$-HCG level per 1SD (OR, 2.73; 95\% CI, 1.13 to $6.60 ; \mathrm{P}=0.026$ ) were independent risk factors for the onset of $\mathrm{PE}$ in twin pregnancies. Primiparous women who have used IVF conception methods had a 7-fold increased risk of PE during twin pregnancy. For every $\beta$-HCG level standard deviation elevation, the risk of $\mathrm{PE}$ increased 2.73 times $(\mathrm{P}<0.05)$. For UTPI (OR, 0.17; 95\% CI, 0.031 to $0.944 ; \mathrm{P}=0.043)$, and every 1.0 increase reduced the risk of PE by $83 \%(\mathrm{P}<0.05)$. Table-III.

The prediction accuracy of using clinical risk factors (parity + conception method), biomarkers, and the UTPI together to screen women with twin pregnancy for PE is shown in the ROC curve in Fig.2; the area under the ROC curve, sensitivity, and specificity are shown in Table-IV. By adding biomarkers and UTPI, the screening performance of the clinical risk factors for twin PE was improved. When the false positive rate (FPR) is $5 \%$, using a combination of clinical risk factors, serum markers, and UTPI, increases the prediction accuracy for PE during twin pregnancies based on an AUC of 0.928, sensitivity of $85 \%$, and specificity of $88 \%$.

\section{DISCUSSION}

This study examined the ability of various clinical risk factors and biomarkers to predict PE in women with twin pregnancy. It found four independent predictors for PE onset during twin
Table-III: Multivariate Logistic regression analysis of the two groups.

\begin{tabular}{lccc}
\hline Parameter & Or & $95 \% c i$ & P value \\
\hline Parity & 7.51 & $1.04-54.18$ & 0.045 \\
$\begin{array}{l}\text { Conception method } \\
\text { B-HCG(mIU/ml) }\end{array}$ & 7.11 & $1.14-44.36$ & 0.036 \\
(per 1 SD) & 2.73 & $1.13-6.60$ & 0.026 \\
UTPI & 0.17 & $0.031-0.944$ & 0.043 \\
Age & 2.537 & $0.156-41.292$ & 0.513 \\
\hline
\end{tabular}

pregnancy: first delivery, conception method, $\beta$-HCG level, and UTPI. Predictive power, as well as sensitivity and specificity, improved when multiple factors were combined.

The onset of PE is earlier for women with twin pregnancy, and is associated with higher maternal and perinatal mortality.5,6 Therefore, early prediction and detection of PE are essential to improve outcomes. Current guidelines recommend continuous low-dose $(75-162 \mathrm{mg} / \mathrm{d})$ aspirin to people at high risk of $\mathrm{PE}$ between 16 to 36 weeks of gestation, to prevent PE and improve maternalfetal pregnancy outcomes. ${ }^{7-9}$ However, due to the side effects of the drug, not every woman with a twin pregnancy should take aspirin, and usually compliance is low. ${ }^{10}$ Developing early PE prediction methods for twin pregnancies will allow timely identification of women with twin pregnancies who would benefit from the treatment to avoid nonselective aspirin administration.

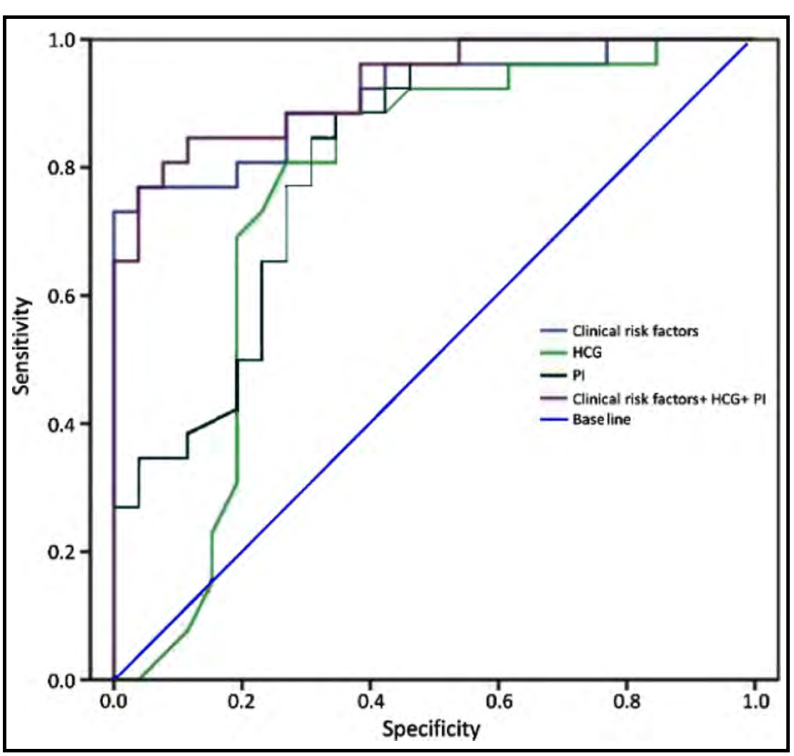

Fig.2: ROC of each variable to predict preeclampsia of twin individually and jointly. 
Table-IV: The value of each factor for independent or joint PE prediction during twin pregnancies.

\begin{tabular}{lccccc}
\hline Parameter & Sensitivity & Specificity & AUC & 95\%CI & P value \\
\hline Clinical risk factors (parity + method of conception) & 69 & 92 & 0.837 & $0.726+0.947$ & $<0.001$ \\
$\beta$-HCG & 81 & 78 & 0.8 & $0.677-0.924$ & $<0.001$ \\
UTPI & 88 & 65 & 0.814 & $0.697-0.930$ & $<0.001$ \\
Clinical risk factors + $\beta$-HCG+UTPI & 85 & 88 & 0.928 & $0.860-0.995$ & $<0.001$ \\
\hline
\end{tabular}

Recent studies suggest a two-stage model of PE pathogenesis: placental superficial implantation followed by placental oxidative stress and inflammation. ${ }^{8}$ The incidence of PE in twin pregnancies may also be associated with maternal factors, such as high body mass index (BMI) and high blood coagulation tendency. ${ }^{11,12} \mathrm{~A}$ study by Taguchi et al. ${ }^{13}$ on 742 women with twin pregnancies found that first-time delivery, high BMI, family history of hypertension, and history of hypertension during a previous pregnancy were all significant risk factors for PE with ORs at 1.77 (95\% CI, 1.21 to 2.61$), 1.35$ (95\% CI, 1.08 to 1.70$), 1.50$ (95\% CI, 1.02 to 2.17$)$, and 8.85 (95\% CI, 2.70 to 29.0), respectively. Benko et al. ${ }^{14}$ used maternal factor information from 2219 women with twin pregnancy as a training data set to build a PE prediction model, tested data from 2999 women with twin pregnancy, and reported the outcome of early onset PE prediction. The AUCs for all PE cases were 0.670 (95\% CI, 0.593-0.747) and 0.677 ( $95 \% \mathrm{CI}, 0.594$ to 0.760 ), the predicted AUCs for all PE cases were 0.656 (95\% CI, 0.615 to 0.697 ) and 0.644 (95\% CI, 0.606 to 0.682 ).

Studies show that the frequency of PE during twin pregnancies is two to three times higher than during singleton pregnancies, and the incidental rate ranges from $13 \%$ to $26 \%{ }^{15,16}$ Consistently with these reports, we found that $17 \%$ of women with twin pregnancy in our cohort developed PE. The results of our multivariate logistic analysis indicated that first-time delivery pregnancy and IVF conception methods were significantly associated with PE during twin pregnancies. Both first-time delivery (OR, 7.51; 95\% CI, 1.04 to 54.18; $\mathrm{P}=0.045)$ and conception method (OR, $7.11 ; 95 \%$ $\mathrm{CI}, 1.14$ to $44.36 ; \mathrm{P}=0.036)$ were associated with a 7 -fold increase in PE risk. The false positive rate (FPR) was 5\%, and the detection rate of PE during twin pregnancies was $69 \%$.

PE has been linked to vascular and endothelial abnormalities. During normal pregnancies, thickness of the inner diameter of the uterine artery increases to meet the changing demands in placental blood supply, and the blood flow switches from its normal state of high resistance and low discharge to a pregnant state of low resistance and high discharge. As the pregnancy progresses, the uterine artery blood flow resistance drops significantly, and the UTPI gradually decreases. In patients with PE, number of remodeling uterine spiral arteries is reduced, due to the incomplete erosion by trophoblast cells. This results in relatively high vascular resistance, while the uterine artery blood flow is characterized by high resistance and low discharge ${ }^{17}$ accompanied by significantly elevated UTPI. Uterine artery blood flow parameters have become a common method to predict the risk of PE during singleton pregnancies. ${ }^{18}$ The consensus states that in both singleton and twin pregnancies, the uterine artery blood flow resistance is decreased with increase in gestational age, and that UTPI during twin pregnancies is consistently lower than that of singleton pregnancies. ${ }^{19,20}$ However, there are only few inconclusive studies on the association between UTPI levels in women with twin pregnancy and PE. Lack of consensus may be due to UTPI measurements done at different gestational weeks. ${ }^{21,22}$ We found that the mean UTPI in women with twin pregnancy and PE was lower than in women without PE. UTPI in the twin PE group (OR, 0.17; 95\% CI, 0.031 to 0.944; $\mathrm{P}=0.043$ ) suggests that every UTPI increase by one reduces the risk of PE by $83 \%$. When the FPR is $5 \%$, the AUC for PE is 0.814 with UTPI alone, and when the sensitivity is $88 \%$, the specificity is $65 \%$. For women with twin pregnancy and PE, abnormal blood supply of one placenta may cause a compensatory increase in the blood supply to the other placenta. ${ }^{23}$ Due to the limited sample size in our study, we did not analyze the UTPI characteristics of women with identical twins and fraternal twins and PE. The mechanisms leading to abnormal UTPIs in women with twin pregnancy and PE need to be studied. 
Many studies focused on serum biomarkersin general and $\beta$-HCG in particular, as predictors and prognostic factors of PE. ${ }^{24,25}$

$\beta$-HCG is mostly synthesized by placental trophoblastic cells, which are important indicators of placental function. ${ }^{26}$ However, few studies have focused on the changes in serum $\beta$-HCG in women with twin pregnancies and PE. Metz et al. ${ }^{27}$ found that the serum $\beta$-HCG level during the first trimester of twin pregnancies is higher than that during singleton pregnancies, and that the serum $\beta$-HCG level in people with twin pregnancies that go on to develop PE is significantly higher than that of women without PE $(P=0.004)$. Iskender et $a{ }^{28}$ confirmed that the serum $\beta$-hCG level in women with twin pregnancies and PE was higher than in women with normal twin pregnancies, and that a high $\beta$-hCG level was associated with adverse pregnancy outcomes such as fetal growth restriction. Our results show that everyone standard deviation from the normal $\beta$-HCG levels increases risk of PE 2.73-fold (OR,2.73; 95\% CI, 1.13 to 6.60; $\mathrm{P}=0.026)$. When the FPR is $5 \%$, the AUC in women with twin pregnancy and $\mathrm{PE}$ alone is 0.800 , and when the sensitivity is $81 \%$, the specificity is $78 \%$.

Study Limitations: The study has two main limitations. The first is a small sample size, which prevented us from investigating certain relationships and associations, such as UTPI characteristics of women with PE who bore identical twins vs. those who bore fraternal twins. The second is that the study population was relatively homogenous, since all patient data was collected through a single regional health facility. Additional investigations on larger and more heterogeneous study populations will need to be performed to further evaluate the predictive power of the highlighted parameters and biomarkers.

\section{CONCLUSION}

In summary, this study demonstrated that combining early pregnancy serum markers $(\beta$-HCG) and UTPI markedly improves their sensitivity, specificity, and overall predictive value for PE during twin pregnancy. Future studies with large sample sizes and long follow-ups are needed to establish a PE predictive model for twin pregnancies that has a low false positive rate and a high detection rate. The chosen model should improve the early diagnostic efficiency for PE during twin pregnancies to provide a reliable diagnostic basis for effective treatments and interventions.
Funding: We received funding from the Public Welfare Technology Research Project of the Huzhou Science and Technology Bureau (grant number 2019GYB15) and the Zhejiang Province Medical Science and Technology Plan (grant number 2021KY1084).

Availability of data and materials: The datasets used and/or analyzed during the current study are available from the corresponding author on reasonable request.

Competing interests: None.

\section{REFERENCES}

1. Ngoc NTN, Merialdi M, Abdel-Aleem H, Carroli G, Purwar $\mathrm{M}$, Zavaleta N, et al. Causes of stillbirths and early neonatal deaths: data from 7993 pregnancies in six developing countries. Bull World Health Organ. 2006;84(9):699-705. doi: $10.2471 /$ blt.05.027300

2. Francisco C, Wright D, Benkő Z, Syngelaki A, Nicolaides $\mathrm{KH}$. Hidden high rate of pre-eclampsia in twin compared with singleton pregnancy. Ultrasound Obstet Gynecol. 2017;50(1):88-92. doi: 10.1002/uog. 17470

3. Hypertension in pregnancy. Report of the American College of Obstetricians and Gynecologists' Task Force on Hypertension in Pregnancy. Obstet Gynecol. 2013;122(5):1122-1131. doi: 10.1097/01. AOG.0000437382.03963.88

4. Sotiriadis A, Hernandez-Andrade E, da Silva Costa F, Ghi T, Glanc P, Khalil A, et al. ISUOG Practice Guidelines: role of ultrasound in screening for and follow-up of preeclampsia. Ultrasound Obstet Gynecol. 2019;53(1):7-22. doi: 10.1002/uog. 20105

5. Sibai BM, Hauth J, Caritis S, Lindheimer MD, MacPherson C, Klebanoff M, et al. Hypertensive disorders in twin versus singleton gestations. National Institute of Child Health and Human Development Network of Maternal-Fetal Medicine Units. Am J Obstet Gynecol. 2000;182(4):938-942. doi: 10.1016/s0002-9378(00)70350-4

6. Fox NS, Roman AS, Saltzman DH, Hourizadeh T, Hastings J, Rebarber A. Risk factors for preeclampsia in twin pregnancies. Am J Perinatol. 2014;31(2):163-166. doi: 10.1055/s-0033-1343775

7. Sentilhes L, Azria E, Schmitz T. Aspirin versus Placebo in Pregnancies at High Risk for Preterm Preeclampsia. N Engl J Med. 2017;377(24):2399-2400. doi: 10.1056/NEJMc1713798

8. Good clinical practice advice: First trimester screening and prevention of pre-eclampsia in singleton pregnancy. Int J Gynecol Obstet. 2019;144(3):325-329. doi: 10.1002/ijgo.12741

9. Brown MA, Magee LA, Kenny LC, Karumanchi SA, McCarthy FP, Saito S, et al. Hypertensive Disorders of Pregnancy: ISSHP Classification, Diagnosis, and Management Recommendations for International Practice. Hypertension. 2018;72(1):24-43. doi: 10.1161/ HYPERTENSIONAHA.117.10803

10. Sibai BM, Caritis SN, Thom E, Klebanoff M, McNellis D, Rocco L, et al. Prevention of preeclampsia with lowdose aspirin in healthy, nulliparous pregnant women. The National Institute of Child Health and Human Development Network of Maternal-Fetal Medicine Units. N Engl J Med. 1993;329(17):1213-1218. doi: 10.1056/ NEJM199310213291701 
11. Wolf M, Shah A, Lam C, Martinez A, Smirnakis KV, Epstein $\mathrm{FH}$, et al. Circulating levels of the antiangiogenic marker sFLT-1 are increased in first versus second pregnancies. Am J Obstet Gynecol. 2005;193(1):16-22. doi:10.1016/j. ajog.2005.03.016

12. Faupel-Badger JM, Staff AC, Thadhani $R$, Powe $C E$, Potischman N, Hoover RN, et al. Maternal angiogenic profile in pregnancies that remain normotensive. Eur J Obstet Gynecol Reprod Biol. 2011;158(2):189-193. doi: 10.1016/j.ejogrb.2011.05.001

13. Taguchi T, Ishii K, Hayashi S, Mabuchi A, Murata M, Mitsuda N. Clinical features and prenatal risk factors for hypertensive disorders in twin pregnancies. J Obstet Gynaecol Res. 2014;40(6):1584-1591. doi: 10.1111/jog.12408

14. Benko Z, Chaveeva P, de Paco Matallana C, Zingler E, Wright A, Wright $\mathrm{D}$, et al. Validation of competing-risks model in screening for pre-eclampsia in twin pregnancy by maternal factors. Ultrasound Obstet Gynecol. 2019;53(5):649-654. doi: 10.1002/uog.20265

15. American College of Obstetricians and Gynecologists Committee on Practice Bulletins-Obstetrics, Society for Maternal-Fetal Medicine, ACOG Joint Editorial Committee. ACOG Practice Bulletin \#56: Multiple gestation: complicated twin, triplet, and high-order multifetal pregnancy. Obstet Gynecol. 2004;104(4):869-883. doi: 10.1097/00006250-200410000-00046

16. Krotz S, Fajardo J, Ghandi S, Patel A, Keith LG. Hypertensive disease in twin pregnancies: a review. Twin Res. 2002;5(1):8-14.

17. Shah DA, Khalil RA. Bioactive factors in uteroplacental and systemic circulation link placental ischemia to generalized vascular dysfunction in hypertensive pregnancy and preeclampsia. Biochem Pharmacol. 2015;95(4):211-226. doi:10.1016/j.bcp.2015.04.012

18. Napolitano R, Rajakulasingam $R$, Memmo A, Bhide A, Thilaganathan B. Uterine artery Doppler screening for pre-eclampsia: Comparison of the lower, mean and higher first-trimester pulsatility indices. Ultrasound Obstet Gynecol. 2011;37(5):534-537. doi: 10.1002/uog.8848

19. Yu CKH, Papageorghiou AT, Boli A, Cacho AM, Nicolaides KH. Screening for pre-eclampsia and fetal growth restriction in twin pregnancies at 23 weeks of gestation by transvaginal uterine artery Doppler. Ultrasound Obstet Gynecol. 2002;20(6):535-540. doi: 10.1046/j.14690705.2002.00865.x

20. Geipel A, Berg C, Germer U, Katalinic A, Krapp M, Smrcek $\mathrm{J}$, et al. Doppler assessment of the uterine circulation in the second trimester in twin pregnancies: prediction of pre-eclampsia, fetal growth restriction and birth weight discordance. Ultrasound Obstet Gynecol. 2002;20(6):541545. doi: 10.1046/j.1469-0705.2002.00866.x
21. Rizzo G, Pietrolucci ME, Aiello E, Capponi A, Arduini D. Uterine artery Doppler evaluation in twin pregnancies at $11+0$ to $13+6$ weeks of gestation. Ultrasound Obstet Gynecol. 2014;44(5):557-561. doi: 10.1002/uog.13340

22. Svirsky R, Yagel S, Ben-Ami I, Cuckle H, Klug E, Maymon R. First trimester markers of preeclampsia in twins: maternal mean arterial pressure and uterine artery Doppler pulsatility index. Prenat Diagn. 2014;34(10):956960. doi: 10.1002/pd.4402

23. Hubinont C, Lewi L, Bernard P, Marbaix E, Debiève F, Jauniaux E. Anomalies of the placenta and umbilical cord in twin gestations. Am J Obstet Gynecol. 2015;213(4):S91-S102. doi: 10.1016/j.ajog.2015.06.054

24. Kasraeian M, Asadi N, Vafaei H, Zamanpour T, Shahraki HR, Bazrafshan K. Evaluation of serum biomarkers for detection of preeclampsia severity in pregnant women. Pak J Med Sci. 2018;34(4):869-873. doi:10.12669/pjms.344.14393

25. Ozdamar O, Gun I, Keskin U, Kocak N, Mungen E. The role of maternal serumbeta-HCG and PAPP-A levels at gestational weeks 10 to 14 in the prediction of preeclampsia. Pak J Med Sci. 2014;30(3):568-573. doi:10.12669/ pjms.303.4554

26. Kavak ZN, Basgul A, Elter K, Uygur M, Gokaslan H. The efficacy of first-trimester PAPP-A and free beta hCG levels for predicting adverse pregnancy outcome. J Perinat Med. 2006;34(2):145-148. doi: 10.1515/JPM.2006.026

27. Metz TD, Allshouse AA, Euser AG, Heyborne KD. Preeclampsia in high risk women is characterized by risk group-specific abnormalities in serum biomarkers. Am J Obstet Gynecol. 2014;211(5):512.e1-512.e6. doi: 10.1016/j. ajog.2014.04.027

28. Iskender C, Tarım E, Çok T, Yalcınkaya C, Kalaycı H, Yanık FB. Obstetrical complications associated with first-trimester screening markers in twin pregnancies. J Obstet Gynaecol Res. 2013;39(11):1495-1499. doi: 10.1111/ jog.12091

\section{Authors' Contributions:}

YL: Conceived and designed the study.

ZD, WL, LM, LS, HS \& YL: Collected the data and performed the analysis.

YL: Prepared the manuscript.

YL: Was responsible for the integrity of the study.

All authors have read and approved the final manuscript.

\footnotetext{
Authors:

1. Dr. Yan Lu,

2. Dr. Zhongying Ding,

3. Dr. Wenwen Li,

4. Dr. Lina Mei,

5. Dr. Linglong Shen,

6. Dr. Huaying Shan,

1-6: Department of Obstetrics and Gynecology, Huzhou Maternity and Child Health Care Hospital, Huzhou 313000, Zhejiang Province, P.R. China.
} 